\title{
A quality improvement project to address the challenges surrounding zoledronic acid use in children
}

\author{
David R. Weber ${ }^{1,3}$ (1) Maria Cristina Gil Diaz ${ }^{1} \cdot$ Silvia Louis $^{1} \cdot$ Noya Rackovsky ${ }^{1} \cdot$ Roman Rahmani $^{1,4}$. \\ Sierra D. Stauber ${ }^{1,2}$
}

Received: 1 October 2020 / Accepted: 10 February 2021 / Published online: 7 April 2021

(c) The Japanese Society Bone and Mineral Research 2021

\begin{abstract}
Introduction Zoledronic acid (ZA) is an intravenous bisphosphonate used to treat pediatric osteoporosis. Adverse events including hypocalcemia and acute phase reaction (APR) are common following first-infusion. The purpose of this report is to describe implementation of a ZA clinical practice guideline and the subsequent process changes to improve adherence to aspects of the protocol related to safety and efficacy.

Methods Quality assurance was evaluated by chart review over a 5-year period to compare the prevalence of hypocalcemia and APR to published data. A quality improvement (QI) initiative consisting of process changes including the addition of an endocrine RN to coordinate infusions and a shift to patient/family self-scheduling of infusions was conducted. The effect of the interventions on safety (completion of pre- and post-infusion bloodwork) and efficacy (receipt of all prescribed infusions) outcomes was evaluated.

Results Seventy-two patients received 244 infusions over the period. The frequency of hypocalcemia (22\%) and APR $(31 \%)$ was consistent with prior reports. $99 \%$ of patients received pre-infusion bloodwork, $78 \%$ received post-first-infusion bloodwork, and $47 \%$ received all prescribed infusions. QI initiatives increased the percentage of patients receiving postfirst-infusion bloodwork from 67 to $79 \%$ and those receiving all infusions from 62 to $74 \%$, but fell short of the goal of $90 \%$. Conclusions The implementation of a standardized protocol for ZA use in children was successful in confirming patient eligibility with pre-infusion bloodwork but failed to ensure that patients obtained post-first-infusion bloodwork and received all prescribed infusions. Further efforts to systematize the management of children on ZA are needed.
\end{abstract}

Keywords Zoledronic acid · Bisphosphonates · Osteoporosis · Quality improvement · Quality assessment

David R. Weber

weberd@email.chop.edu

1 Department of Pediatrics, Golisano Children's Hospital, University of Rochester Medical Center, 601 Elmwood Avenue, Box 590, Rochester, NY, USA

2 Department of Pharmacy, University of Rochester Medical Center, 601 Elmwood Avenue, Box 590, Rochester, NY, USA

3 Present Address: Department of Pediatrics, The Children's Hospital of Philadelphia, Roberts Center for Pediatric Research, 2716 South Street, Philadelphia, PA, USA

4 Present Address: Mercy Health - St. Vincent Medical Center, Toledo, OH, USA

\section{Introduction}

Bisphosphonates are anti-osteoclastic pharmacologic agents used to treat disorders of bone and mineral metabolism including osteoporosis, hypercalcemia, and bone pain [1]. Large clinical trials have confirmed the safety and efficacy of bisphosphonates in adults [2]. High quality clinical trial data are lacking for pediatric populations, however, due to factors including the lower prevalence of severe bone disease and greater heterogeneity in the origin of disordered bone and mineral metabolism in children. As a result, clinical bisphosphonate therapy in children has largely been guided by observational studies and expert opinion [3, 4]. Intravenous (IV) formulations appear to have greater efficacy in children and are, therefore, more widely used than oral formulations [5]. 
Zoledronic acid (ZA) is the most potent IV bisphosphonate, and has gained favor in pediatric use because of its short infusion time (typically $30 \mathrm{~min}$ ) and reasonable dosing interval (generally every 6-12 months). Complications of ZA therapy include hypocalcemia (and sequelae, including tetany, seizure), hypophosphatemia (usually asymptomatic), acute kidney injury (if pre-existing renal insufficiency), and an acute phase reaction (APR, fever, myalgia, nausea, vomiting, diarrhea). These side effects typically develop within 48-72 $\mathrm{h}$ after the infusion and are more common and intense with the first infusion. The severity of adverse reactions can be mitigated with appropriate patient selection, provision of calcium \pm calcitriol (active vitamin D), and antipyretics. In rare cases, the reactions can be severe and lead to organ dysfunction [6].

Several centers have reported retrospective analyses investigating the safety and efficacy of ZA in clinical pediatric practice $[7,8]$. The rates of ZA related side effects, effects on bone density and fracture in these reports have varied widely [9]. Non-standardization of ZA dosing and heterogeneity in patient populations may explain some of these differences. Inconsistent adherence to treatment plans is another factor that is not often discussed but has the potential to increase risk and decrease efficacy of ZA infusions. ZA treatment protocols tend to be time intensive, in many cases requiring pre-infusion screening blood-work, travel to a referral center for IV infusion, and post-infusion monitoring bloodwork. Moreover, many of the children prescribed ZA are medically complex and factors such as intercurrent illness and time competition with other medical specialty visits may interrupt or delay prescribed treatment courses.

The objective of this report is to describe the implementation of a clinical practice guideline for the use of $\mathrm{ZA}$ in a children's hospital and the subsequent process changes attempted to improve provider and patient adherence to this guideline, especially with regards to elements intended to ensure safety and promote efficacy of therapy.

\section{Materials and Methods}

\section{Setting}

In 2015 we implemented a hospital approved practice guideline for the use of ZA in pediatric patients at the Golisano Children's Hospital, University of Rochester Medical Center (URMC). The goal of the practice guideline (summarized in Supplemental Table 1) was to standardize ZA eligibility, dosing, prophylaxis, monitoring, and treatment of ZA related complications (hypocalcemia, acute phase response). Indication for ZA use at our institution under this protocol included treatment of primary and secondary osteoporosis, perioperative treatment of low bone mineral density for age in patients with underlying conditions placing them at high risk for skeletal fragility undergoing scoliosis repair (spinal muscular atrophy, for example), hypercalcemia, and bone pain associated with avascular necrosis, fibrous dysplasia, or chronic recurrent multifocal osteomyelitis. The protocol was designed such that the majority of infusions would occur in the outpatient Pediatric Treatment Center (PTC). A minority of patients deemed to be at high risk of complications (age less than 2 years, intractable or inadequately controlled epilepsy, respiratory insufficiency requiring supplemental oxygen or mechanical support, cardiac disease) would be admitted to the hospital for the first infusion. Order sets in the electronic health record (EHR) system were developed for use in both the PTC and in the Children's Hospital. The guideline recommended that serum calcium and phosphorus be assessed and confirmed normal within 14 days prior to all infusions, that post-infusion calcium levels be checked 48-72 $\mathrm{h}$ after first infusions, and that vitamin D sufficiency (serum $25-\mathrm{OH}$ vitamin $\mathrm{D} \geq 20 \mathrm{ng} / \mathrm{mL}$ ) be established prior to first infusion and annually thereafter.

A multi-disciplinary team lead by a pediatric endocrinologist and including a clinical pharmacist, inpatient and outpatient nurses, and office administrative staff was involved the development, evaluation, and process changes described in this report. A limited number of patients were treated under this guideline in the first year, under close supervision of the endocrine provider. After the first year, scheduling, pre-, and post-infusion calling of families was delegated to office administrative staff (OAS), based on a work-flow sheet developed jointly by the provider and OAS. A chart review conducted after approximately 12 months suggested that an unsatisfactory number of patients were meeting certain safety and efficacy elements of the protocol, prompting the quality improvement (QI) initiative.

\section{Population and outcome measures}

Clinical data were abstracted from the EHR for all pediatric patients who received ZA infusions between 2015 and 2020 (Supplemental Fig. 1). Patients were identified from a list kept by the provider and checked against pharmacy records. The prevalence of hypocalcemia (serum calcium $<8.5 \mathrm{mg}$ / $\mathrm{dL}$ ), symptomatic hypocalcemia (as documented in chart), signs/symptoms of APR (fever, myalgia as documented in the chart), hospital re-admission, post-infusion emergency department (ED) visits, and discontinuation of ZA prompted by side effects was calculated as means of assessing quality assurance (QA) for comparison to previously published data.

QI outcomes were selected that addressed safety, including: (1) Was ZA eligibility confirmed with required pre-infusion labs (normal calcium and phosphorus within 14 days of infusion), (2) Was vitamin D status confirmed to be adequate in 12 months prior to infusion (serum 25-OH vitamin 
$\mathrm{D} \geq 20 \mathrm{ng} / \mathrm{mL}$ ), (3) Did patients receive recommended laboratory monitoring 48-72 $\mathrm{h}$ after the first infusion (to assess for hypocalcemia), and efficacy: (1) Did patients receive all of the prescribed ZA infusions over the time interval (allowing for up to 3 months of delay. For example, if a patient was placed on an every 6-month dosing schedule, receipt of a second dose within 9 months would qualify as meeting the recommendation).

\section{Interventions}

Two subsequent process changes were implemented and evaluated. Three time periods were included in the QI evaluation (pre-intervention, first follow-up, second follow-up). The evaluation periods were 18 months (the second followup was truncated at 16 months $\mathrm{d} / \mathrm{t}$ COVID pandemic) given the small number of patients on ZA. The goal was to achieve $90 \%$ adherence for each outcome.

- The first process change consisted of four components aimed primarily at the provider team to enhance communication and tracking of patients on ZA therapy:

o Infusion scheduling, along with pre- and post-infusion patient communications was transitioned from OAS to a dedicated endocrine RN.

p RN would review pre- and post-infusion lab results and would alert provider to missing results or for results outside of the protocol parameters

q Patient infusion dates were added to the endocrine provider's calendar by the RN to facilitate review of clinical data

$\mathrm{r}$ A shared ZA patient list was created in the EHR to facilitate tracking

- The second process change was aimed at patients/families and intended to facilitate timely ZA infusion scheduling:

o Patients were asked to schedule the follow-up infusion prior to leaving PTC at current infusion. This process was facilitated by the PTC RN via an order placed in the EHR by the endocrine provider

\section{Ethics}

This project was determined by the URMC Institutional Review Board (IRB) to not meet the definition of human subject research. Per the IRB: "This project was conducted as a Quality Improvement initiative, and as such was not formally supervised by the Institutional Review Board per their policies."

\section{Statistical analysis}

Standard descriptive statistics were used to summarize clinical data. Pre- and post-intervention proportions were compared using chi-square (or Fishers exact if any group contained less than five observations). All analyses were conducted in STATA 16 (StataCorp LLC). The manuscript was drafted to conform with the Squire 2.0 Quality Improvement Reporting guidelines [10].

\section{Results}

Seventy-two patients received a total of 244 ZA infusions over the 5-year evaluation period. The majority (84\%) of infusions occurred in the PTC, including $53 \%$ of first infusions. The majority of patients $(78 \%)$ received a first ZA dose of $0.0125 \mathrm{mg} / \mathrm{kg}$, as per the protocol. Patients who were admitted for infusions were more likely to have secondary osteoporosis compared to those treated in the PTC, but did not differ by age, sex, racial group, or initial ZA dose (Table 1).

\section{QA analyses}

Post-infusion hypocalcemia occurred following 22\% (17/76) of all infusions in which post-infusion labs were obtained. One case of hypocalcemia was documented as symptomatic (tetany). The prevalence of documented hypocalcemia did not differ between first (21\%) and subsequent (25\%) infusions, $p=0.75$. It is important to note, however, that as expected per protocol, the proportion of subsequent infusions with post-infusion lab monitoring for hypocalcemia $(12 \%)$ was dramatically lower vs first infusions (78\%), $p<0.001$. Patients with documented hypocalcemia did not differ from those without hypocalcemia in terms of age, diagnosis, ZA dose, or any pre-infusion laboratory parameter (including calcium, 25-OH vitamin D, alkaline phosphatase). Signs/symptoms of APR occurred following $31 \%$ of all infusions (29/93) in which notes specifically mentioned presence/absence of APR. The prevalence of documented APR did not differ between first (34\%) vs subsequent (26\%) of infusions, $p=0.43$; noting that presence/absence of APR was documented in a greater proportion of first $(86 \%)$ vs subsequent $(18 \%), p<0.001$.

The prevalence of hypocalcemia following first infusion was non-significantly greater in admitted patients vs those treated in the PTC (29 vs 9\%, $p=0.07)$, noting that postfirst-infusion labs were obtained in a greater percentage of admitted vs PTC patients ( 100 vs $58 \%, p<0.001)$. The frequency of reported APR did not differ by location. Two patients ( $<1 \%$ of infusions) were found to have unplanned 
Table 1 Characteristics of 72 patients receiving 244 zoledronic acid infusions

\begin{tabular}{|c|c|c|c|c|}
\hline & All infusions & Inpatient infusions $^{1}$ & Outpatient infusions $^{1}$ & $p$ \\
\hline \multicolumn{5}{|l|}{ Demographics } \\
\hline Unique patients, $n$ & 72 & 34 & 62 & \\
\hline Infusions, $n$ & 244 & 40 & 204 & \\
\hline Male sex, $n^{2}$ & $47(65)^{3}$ & $20(59)^{3}$ & $41(66)^{3}$ & 0.48 \\
\hline Racial Group, $n^{2}$ & & & & 0.60 \\
\hline White & $55(77)$ & $25(74)$ & $51(82)$ & \\
\hline Black & $11(15)$ & $6(18)$ & $7(11)$ & \\
\hline Other & $6(8)$ & $3(8)$ & $4(7)$ & \\
\hline Age at first infusion, years & $11.0(0.2-21.2)^{4}$ & $9.9(0.9-21.2)^{4}$ & $11.5(0.2-20.1)^{4}$ & 0.16 \\
\hline Dose at first infusion, $\mathrm{mg} / \mathrm{kg}$ & $0.0125(0.0125-0.025)^{4}$ & $0.0125(0.0125-0.025)^{4}$ & $0.0125(0.0125-0.025)^{4}$ & 0.10 \\
\hline Average dose, all infusions, $\mathrm{mg} / \mathrm{kg}$ & $0.025(0.0125-0.05)^{4}$ & $0.0125(0.0125-0.025)^{4}$ & $0.025(0.0125-0.05)^{4}$ & $<0.01$ \\
\hline Diagnosis, $n^{2}$ & & & & 0.05 \\
\hline Osteogenesis imperfecta $^{5}$ & $21(29)$ & $5(15)$ & $20(32)$ & \\
\hline Secondary osteoporosis & $36(50)$ & $24(70)$ & $28(45)$ & \\
\hline Other & $15(21)$ & $5(15)$ & $14(23)$ & \\
\hline \multicolumn{5}{|l|}{ Quality assurance outcomes } \\
\hline Hypocalcemia $^{6}$ & $17 / 76(22)$ & $13 / 40(32)$ & $4 / 36(11)$ & 0.03 \\
\hline Symptomatic & $1 / 76(1)$ & $1 / 40(3)$ & $0 / 36(0)$ & 0.34 \\
\hline Acute phase reaction ${ }^{7}$ & $29 / 93(31)$ & $14 / 40(35)$ & $15 / 53(28)$ & 0.49 \\
\hline Emergency department visit & $2 / 244(1)$ & $0 / 40(0)$ & $2 / 204(1)$ & 0.89 \\
\hline Therapy stopped due to side effects & $1 / 244(<1)$ & $1 / 40(3)$ & $0 / 204$ & 0.03 \\
\hline Length of stay, hours & N/A & $48(45.5-72.5)^{8}$ & N/A & N/A \\
\hline \multicolumn{5}{|l|}{ Quality improvement outcomes } \\
\hline Pre-infusion labs obtained & 236/244 (99) & 40/40 (100) & $200 / 204(98)$ & 0.37 \\
\hline Vitamin D sufficiency established ${ }^{9}$ & $228 / 244(93)$ & $37 / 40(93)$ & $191 / 204(94)$ & 0.79 \\
\hline Post-first-infusion labs obtained & $56 / 72(78)$ & $34 / 34(100)$ & $22 / 38(58)$ & $<0.01$ \\
\hline Received all prescribed infusions & $34 / 72(47)$ & $9 / 34(26.5)^{10}$ & $25 / 38(66)$ & $<0.01$ \\
\hline
\end{tabular}

${ }^{1}$ Includes all patients with at least one inpatient or outpatient infusion, respectively. Patients may contribute data to both categories if infusions were received in both locations, unless otherwise noted. $p$ is the comparison for inpatient vs outpatient

${ }^{2}$ Calculated in terms of unique patients

${ }^{3} n(\%)$, all such values not otherwise noted

${ }^{4}$ Median (range)

${ }^{5}$ Includes other rare genetic bone disorders with low bone density and fractures

${ }^{6}$ Calculated for all infusions with documented post-infusion blood-work, denominator provided

${ }^{7}$ Calculated for all infusions, where the presence or absence of acute phase reaction documented in chart, denominator provided

${ }^{8}$ Median (interquartile range)

${ }^{9}$ Documentation of serum $25-\mathrm{OHD} \geq 20 \mathrm{ng} / \mathrm{mL}$ in 12 months prior to infusion

${ }^{10}$ Denominator based upon location of first infusion

ED evaluations following the infusion for APR symptoms. Both had normal electrolytes and were discharged without admission. Discontinuation of ZA therapy was attributed to side-effects (seizure during infusion) in one patient ( $1 \%$ of treated patients).

\section{QI analyses}

Analyses of pre-intervention data (33 patients, 64 infusions; including 24 first infusions) revealed that $100 \%$ of patients had acceptable pre-infusion bloodwork, $98 \%$ had documentation of vitamin D sufficiency in 12 months prior to infusion, $67 \%$ had received post-first-infusion bloodwork, and $62 \%$ had received all prescribed infusions. The effect of sequential processes changes on outcomes is shown in Fig. 1. The proportion of patients receiving 


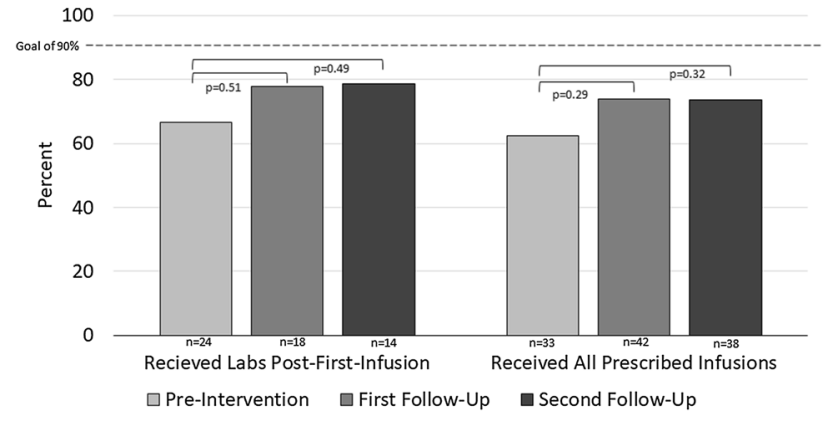

Fig. 1 Effect of sequential quality improvement initiatives on adherence to safety and efficacy outcomes in children on zoledronic acid therapy. The percentage of patients that received post-first-infusion bloodwork (to assess for hypocalcemia) within $72 \mathrm{~h}$ of infusion increased non-significantly from $67 \%$ to a maximum of $79 \%$ after the second intervention, the percentage of patients receiving all prescribed zoledronic acid infusion increased non-significantly from $62 \%$ to a maximum of $74 \%$ after the first intervention. The percentage of patients that had documentation of acceptable pre-infusion bloodwork and vitamin D sufficiency remained $>90 \%$ across the entire time period. $n$ denotes denominator for each time period

post-first-infusion blood-work increased non-significantly after the first intervention (42 patients, 86 infusions; including 18 first infusions) to $78 \%(p=0.51)$ and then again to $79 \%$ ( $p=0.49$ compared to baseline) after the second intervention (38 patients, 71 infusions; including 14 first infusions). The proportion of patients that received all prescribed infusions increased non-significantly to $74 \%$ after the first intervention $(p=0.32)$ and did not change further after the second intervention. The proportion of patients with acceptable pre-infusion bloodwork and with documented vitamin D sufficiency remained $>90 \%$ for both post-intervention time periods.

Collectively over the entire 5-year study period, $99 \%$ of patients had acceptable pre-infusion bloodwork prior to all infusions, 93\% had vitamin D sufficiency documented in every year an infusion was administered, $78 \%$ had postfirst-infusion bloodwork, and $47 \%$ received all prescribed infusions. $36 \%$ of patients had post-first-infusion bloodwork and received all infusions, $8 \%$ had neither bloodwork nor received all infusions, and $56 \%$ had one or the other. The six patients who met neither criteria were older than those who met both or one criterion [17.9 (IQR: 15.6-19.6) vs 10.9 (IQR: 5.5-14.9) vs 10.4 (IQR: 7.4-14), $p=0.02$ ], but did not differ by diagnosis or any other criteria. Patients with secondary osteoporosis were less likely to have received all prescribed infusions compared to other patients (33 vs $61 \%$, $p=0.02$ ). Patients who were admitted for at least one ZA infusion were also less likely to have received all infusions vs those treated solely in the PTC (32 vs $61 \%, p=0.02$ ). Patients with and without post-infusion bloodwork did not differ by any characteristics.

\section{Discussion}

To our knowledge, this is the first QA/QI report describing efforts to improve adherence to an institutional practice guideline for the use of ZA in children. Our protocol was successful in confirming eligibility of patients for ZA infusion through documentation and review of pre-infusion bloodwork. These tasks can be performed with the patient physically present and suggest that both inpatient and outpatient teams were able to understand and adhere to the guidelines in the order-set. Our approach was less successful in ensuring that patients obtained follow-up bloodwork 48-72 $\mathrm{h}$ after first infusions and that patients received the full prescribed ZA treatment course.

Process changes including addition of an endocrine $\mathrm{RN}$ to the care team and a shift to encourage in-person scheduling of follow-up ZA infusions led to modest, though statistically insignificant increases in adherence to these outcomes that failed to achieve our target of $90 \%$. We speculate that the effect of these interventions may have been limited by the fact that implementation remained highly dependent on individual actions (i.e., care team to actively maintain and review a patient list, patient/family to follow-through on care team requests). We identified only one prior report of a QI initiative related to ZA [11]. The prior project was completed in an adult infusion center; therefore, comparisons to our findings are limited. However, it is notable that the authors concluded that implementation of a standardized ZA protocol was successful in ensuring that appropriate preinfusion blood work was obtained.

The finding that less than half of patients on ZA therapy received the full prescribed dose over the analysis period was unexpected and concerning. Partial treatment may reduce the benefits of therapy and thereby change the risk/benefit ratio. Because the likelihood of $\mathrm{ZA}$ related adverse events is highest following the first infusion, patients who receive the first dose but fail to receive recommend follow-up doses may be exposed to the majority of the risk of treatment but fail to experience the full benefits. We could find no similar reports from other centers and are, therefore, unable to place these findings in context. Our data suggest that more medically complex patients (i.e., those with secondary osteoporosis and those admitted for first infusion) were at higher risk of incomplete treatment, but these factors need to be explored further. Next steps under consideration at our center include the possibility of systematizing the work-flow through the creation of a "dashboard" in the EHR. Ideally, this system would include a list of all patients on ZA therapy along with due dates of upcoming infusions, labs, and other meaningful clinical outcomes. Further efforts to understand patient/ family barriers to meeting treatment goals are also needed. 
From a QA perspective, the prevalence of documented hypocalcemia (21\%) and APR (34\%) following first ZA infusion in our patients was within the range of what has been previously reported in children: $16-28 \%$ for hypocalcemia and $17-77 \%$ for APR [7, 12-15]. The prevalence of hypocalcemia may have been higher if all patients had received postinfusion bloodwork, but would not be expected to exceed $29 \%$ (the prevalence of hypocalcemia in admitted patients at higher risk). In conjunction with the low prevalence of symptomatic hypocalcemia and ED evaluations, these data suggest that the implementation of our protocol has achieved safety outcomes consistent with the current standard of care.

Based upon the favorable safety outcomes achieved by the current guideline, we have begun to explore ways of reducing the patient/family burden of ZA infusions. This includes the development and implementation of a protocol for in-home ZA infusions administered by a visiting home $\mathrm{RN}$ for patients who have tolerated previous ZA infusions without adverse event. The low documented incidence of hypocalcemia following ZA infusions suggests the possibility that post-infusion lab assessment may not be necessary for all patients receiving their first infusion. However, we were unable to identify specific risk factors for hypocalcemia in this cohort and cannot provide evidence-based guidance as to which patients could safely forgo post-first-infusion bloodwork.

This project had limitations. Ascertainment of outcome data may have been incomplete if patients obtained bloodwork at external labs or sought post-infusion care at outside institutions. URMC is the regional referral center for complex pediatric care; therefore, we think it unlikely that any ZA treated patients would have been evaluated without notification of our team. Outside of temperature, the signs/ symptoms of APR are subjective and were not well documented. The development of a standard APR questionnaire has been discussed but not yet implemented. Collection of this data may be clinically relevant, especially if development of more severe APR symptoms were to be associated with delay or discontinuation of ZA therapy. The reason for missed infusion was not captured and is an important future direction.

\section{Conclusions}

The clinical practice guideline described in this report was found to be effective at preventing severe ZA related adverse events and can serve as a model for clinicians interested in using ZA in children. Our data show that the majority of $\mathrm{ZA}$ infusions in children can be safely administered in an outpatient setting. The incidence of hypocalcemia following first infusions was low, raising the possibility that routine post-first-infusion blood draws may not be necessary in all patients. Larger samples derived from multiple institutions may be needed to more completely define the risk factors for ZA related adverse events. Our findings of inconsistent adherence to treatment regimens highlight the need for further work to standardize and simplify clinical protocols for the use of bisphosphonates in children.

Supplementary Information The online version contains supplementary material available at https://doi.org/10.1007/s00774-021-01214-5.

Funding DRW was supported by NIH grant DK114477; MCGD and RR were supported by the Strong Children's Research Center.

\section{Compliance with ethical standards}

Conflict of interest David R Weber, Maria Cristina Gil Diaz, Silvia Louis, Noya Rackovsky, Roman Rahmani, and Sierra D Stauber declare that they have no conflict of interest.

\section{References}

1. Eghbali-Fatourechi G (2014) Bisphosphonate therapy in pediatric patients. J Diabetes Metab Disord 13:109

2. Fan Q, Wang J (2020) The efficacy and safety of bisphosphonates for osteoporosis in women older than 65 years: a meta-analysis. Curr Pharm Des 26:4022-4030

3. Palomo T, Fassier F, Ouellet J, Sato A, Montpetit K, Glorieux FH et al (2015) Intravenous bisphosphonate therapy of young children with osteogenesis imperfecta: skeletal findings during follow up throughout the growing years. J Bone Miner Res 30:2150-2157

4. Simm PJ, Biggin A, Zacharin MR, Rodda CP, Tham E, Siafarikas A et al (2018) Consensus guidelines on the use of bisphosphonate therapy in children and adolescents. J Paediatr Child Health $54: 223-433$

5. Ward LM, Rauch F, Whyte MP, D'Astous J, Gates PE, Grogan D et al (2011) Alendronate for the treatment of pediatric osteogenesis imperfecta: a randomized placebo-controlled study. J Clin Endocrinol Metab 96:355-364

6. Trivedi S, Al-Nofal A, Kumar S, Tripathi S, Kahoud RJ, Tebben PJ (2016) Severe non-infective systemic inflammatory response syndrome, shock, and end-organ dysfunction after zoledronic acid administration in a child. Osteoporos Int 27:2379-2382

7. George S, Weber DR, Kaplan P, Hummel K, Monk HM, Levine MA (2015) Short-term safety of zoledronic acid in young patients with bone disorders: an extensive institutional experience. J Clin Endocrinol Metab 100:4163-4171

8. Nasomyont N, Hornung LN, Wasserman H (2020) Intravenous bisphosphonate therapy in children with spinal muscular atrophy. Osteoporos Int 31:995-1000

9. Bowden SA, Mahan JD (2017) Zoledronic acid in pediatric metabolic bone disorders. Transl Pediatr 6:256-268

10. Ogrinc G, Davies L, Goodman D, Batalden P, Davidoff F, Stevens D (2015) SQUIRE 2.0 (Standards for QUality Improvement Reporting Excellence): revised publication guidelines from a detailed consensus process. J Contin Educ Nurs 46:501-507

11. Ong MWS, Jones L (2017) Improving bisphosphonate infusion monitoring at haematology medical day unit. BMJ Qual Improv Rep. https://doi.org/10.1136/bmjquality.u206586.w4692

12. Munns CF, Rajab MH, Hong J, Briody J, Högler W, McQuade M, Little DG, Cowell CT (2007) Acute phase response and mineral status following low dose intravenous zoledronic acid in children. Bone 41:366-370 
13. Sbrocchi AM, Rauch F, Jacob P, McCormick A, McMillan HJ, Matzinger MA et al (2012) The use of intravenous bisphosphonate therapy to treat vertebral fractures due to osteoporosis among boys with Duchenne muscular dystrophy. Osteoporos Int 23:2703-2711

14. Nasomyont N, Hornung LN, Gordon CM, Wasserman H (2019) Outcomes following intravenous bisphosphonate infusion in pediatric patients: a 7-year retrospective chart review. Bone 121:60-67

15. Nasomyont N, Hornung LN, Wasserman H (2019) Intravenous bisphosphonate therapy in children with spinal muscular atrophy. Osteoporos Int 31:995-1000
Publisher's Note Springer Nature remains neutral with regard to jurisdictional claims in published maps and institutional affiliations. 